\title{
Multi-Electrode Plasma Torches: Motivation for Development and Current State-of-the-Art
}

\author{
J.-L. Marqués, G. Forster and J. Schein*
}

University of the Federal Armed Forces, Munich, Germany

\begin{abstract}
Multi-electrode plasma torches are becoming increasingly popular in the thermal spray community due to their good stability and high power plasma jet even when operated with inert gases. Currently the models in use feature either three cathodes and a single anode or three individual anodes connecting to a single cathode. The motivation for development of these plasma torches is based on the inherent instability of single anode/single cathode systems which leads to fluctuating plasma jets resulting in inhomogeneous particle heating. The use of multi-electrode systems has expanded into the realm of low pressure plasma spraying and vacuum plasma spraying with promising results, while atmospheric plasma spraying results show improved coating quality compared to conventional systems. Current research focuses on the development of numerical process modeling as well as the application of advanced diagnostics for process analysis, opening up opportunities for improvement and process control.
\end{abstract}

\section{INTRODUCTION}

Thermal spray is one of the most potent tools for producing thick coatings. The principle of operation is based on a heat source, which under the influence of a strong gas flow, results in a high velocity hot stream of gas. Particles are inserted into the jet, which are heated and accelerated towards an usually cold substrate where they arrive as splats to build up upon each other to form a coating. Various technologies are currently in use which can be distinguished by the heat source [1]. These range from chemical reactions as in the high velocity oxy-fuel spraying (HVOF) over plasmas generated by electricity to systems like the so-called cold spray or kinetic spray, where the majority of the energy that the particle obtains is kinetic energy. Each technology provides a distinct range of particle velocities and temperatures, thus making each suitable for certain materials and applications (Fig. 1).

A fairly common system is the so called plasma spray technology [2], which, when used at atmospheric pressure, will heat up particles in various sizes ranging from metals to ceramics by passing it through a plasma jet of about $15000 \mathrm{~K}$ at the nozzle exit [3], accelerating these particles to a speed of up to $300 \mathrm{~m} / \mathrm{s}$. The plasma jet is produced using so called plasma torches, consisting of a cathode and an anode which are arranged along a common axis, the cathode usually consisting of a cone shaped piece of tungsten, which points at a hollow tubular copper anode ending in a nozzle. The gas flowing between the cathode and the anode is heated by a moving arc resulting in a hot jet that is pushed out the nozzle. The most used industrial plasma torches of this kind are the Sulzer-metco F4 torch or the Praxair SG 100, a representation of which is shown in Fig. (2).

*Address correspondence to this author at the University of the Federal Armed Forces, Munich, Germany; E-mail: jochen.schein@unibw.de

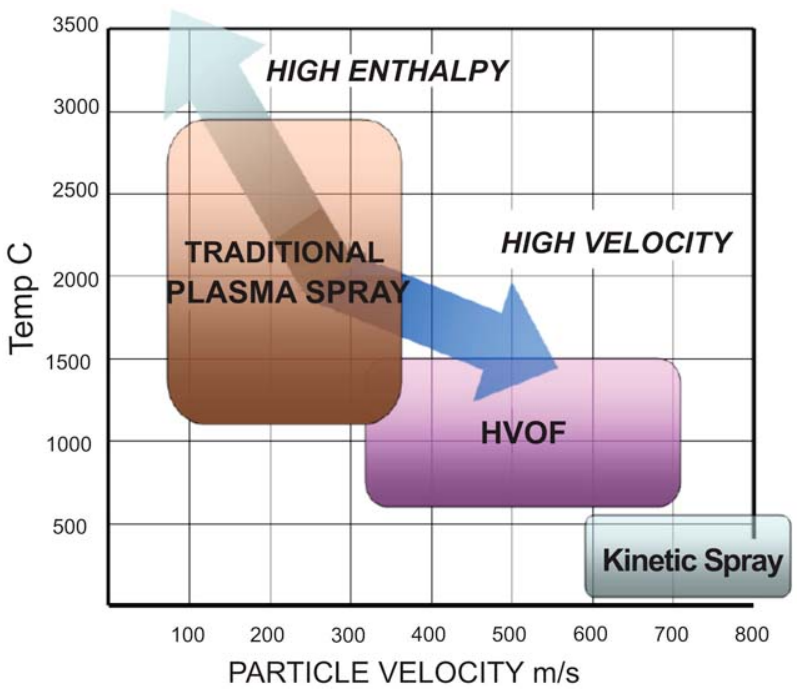

Fig. (1). Temperature range for different applications of thermal spraying [1].

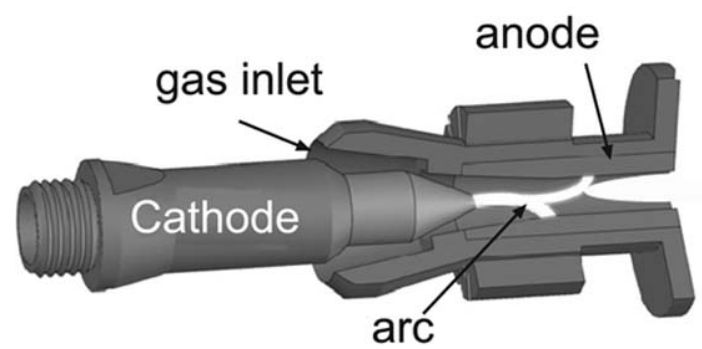

Fig. (2). F4 plasma torch, principle of operation.

Even though plasma spray is an established technology there are still issues that, when solved, could lead to significant improvements of the process. For once the entrainment of the particles into the plasma jet which are 
usually carried by a cold gas flow is still an issue. The most desirable configuration would be a central injection of the particles into the hot gas, which so far has not been realized as the anodic nozzles tend to clog. Thus the particles are inserted at the nozzle exit at angles approaching $90^{\circ}$, with respect to the direction of the main gas flow. However, due to the high viscosity of the plasma jet not all particles enter the hot area, leading to insufficient heating and acceleration and thus less deposition efficiency. In addition the flow, once it leaves the nozzle, becomes turbulent, mixing with the outside atmosphere producing cold islands in the gas flow, which might trap particles [4]. Solution attempts, like the application of shroud gas flows [5] have been described but so far have not led to significant improvements. Another major problem is based on the design of the plasma torches. As can be seen in Fig. (2) the position of the anode attachment can and will change, mostly due to the strong gas flow. This is partly intentional and also enhanced by a vortex component to the axial gas flow as short residence times of the anodic arc root prevent local overheating and melting of the anode surface [6], but it also leads to variations in arc length, and thus to arc voltage/power fluctuations [7, 8]. These fluctuations are also reflected by variations in plasma jet length as high speed images reveal [9], which are shown in Fig. (3) where the change in jet length and luminosity in a frequency range of $\sim 50 \mathrm{kHz}$ is nicely depicted.

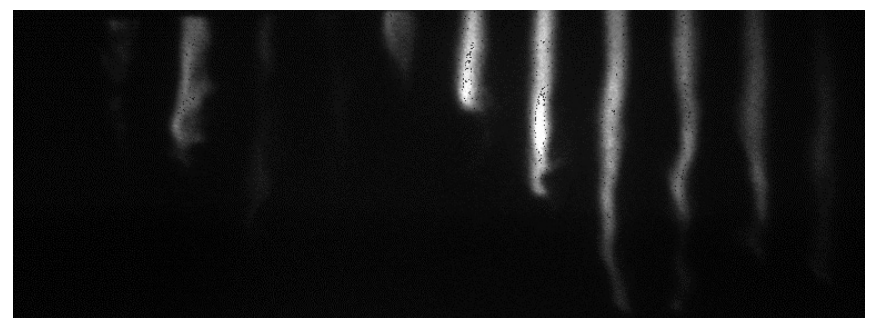

Fig. (3). Fluctuations of a jet generated by a F4 torch, each jet recorded every $20 \mu \mathrm{s}$, exposure time $5 \mathrm{~ns}, 6 \mathrm{~mm}$ nozzle diameter, plasma gas 45/12 slpm $\mathrm{Ar} / \mathrm{N}_{2}$, current $540 \mathrm{~A}$.

Depending on the characteristic processing times $(0.1 \mathrm{~s}-$ $10 \mathrm{~ms}$ ) [10] these variations may lead to fluctuations in particle heating and influence the quality of the coating produced. In addition these torches are limited with respect to gas and power as in order to increase the power in the torch and thus in the jet the plasma torch has to be operated with molecular gas as the jet length cannot be changed in a controlled manner. The operation with gases like $\mathrm{N}_{2}$ or $\mathrm{H}_{2}$ will add the dissociation energy to the plasma enthalpy but in turn constrict the plasma column as well as the arc attachment, leading to a shorter lifetime of the anode. In order to overcome these problems one approach is the design of new plasma torches.

\section{TORCH DEVELOPMENT}

Development of new plasma torches is driven by the desire to improve stability and to increase the range of powders to be used. As early as 1968 developments like the Advanced Plasma Gun (APG) torch (Fig. 4) [11] with a fixed length arc forced by the insertion of neutrodes between the cathode and anode appeared. The long arc length stabilizes the arc by limiting the axial movement thus producing a constant power demand. Similar developments are currently in use with the so-called PJ 100 torch from Plasma Jet Co. [12]. These plasma torches not only produce a more stable jet but make it also easier to adjust the total enthalpy of the plasma gas

$\int_{r=0}^{r=R} \rho(T(r)) v_{\text {axial }}(r) h(T(r)) 2 \pi r d r \approx \dot{W}_{\text {eff }}$

by allowing to adjust the voltage as well as the jet radius by using different gas mixtures. In equation (1) $R$ is the nozzle radius, $\rho$ the gas mass density, $h$ the enthalpy density, $\dot{W}_{\text {eff }}$ the effective electrical torch power (including the loss to the torch cooling) and $v_{\text {axial }}(r)$ and $T(r)$ the radial profile of axial velocity and temperature, both assumed cylindrical symmetric since due to the long neutrode segments the perturbation of the cylinder symmetry only occurs close to the nozzle outlet.

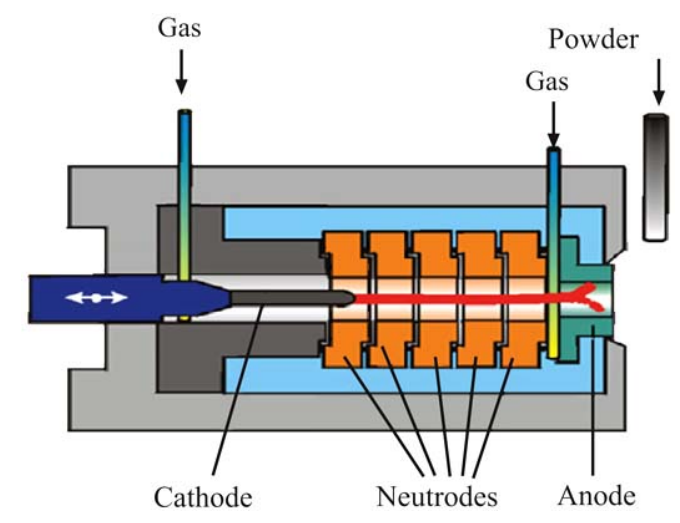

Fig. (4). Schematic of APG plasma torch.

However, inserting the particles into the plasma jet at the nozzle exit always poses a problem. Due to the large angle at which the particles are inserted the gas stream that feeds the particles has to be strong enough to push the particles into the center of the jet while at the same time ideally the hot gas should not be cooled. One way to improve particle feeding is the use of central injection, which in conventional plasma torches would lead to clogging. Thus especially to enable central powder injection multi-electrode systems have been developed [13, 14]. With the advent of nanoparticles and suspensions spray technologies, central injection became even more important for efficient insertion and sufficient heating, thus a closer look at the development of multielectrode plasma torches is justified.

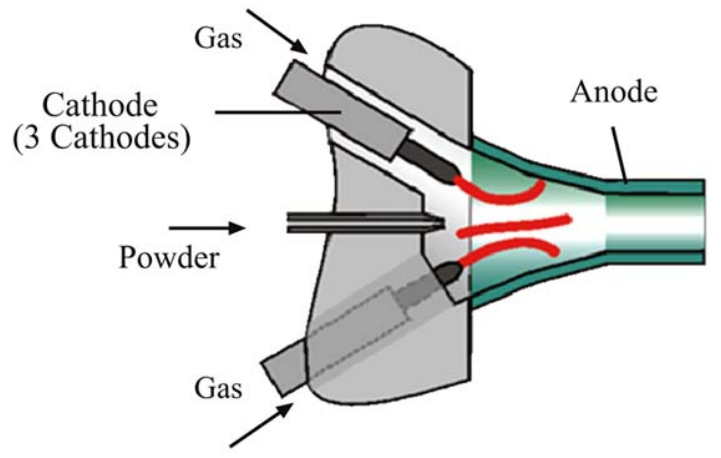

Fig. (5). Multi-electrode torch with three seperated arcs. 
The concept of converging plasma jets about a common axis was probably introduced by a Japanese patent application in 1985 by Fukanuma [15]. In this system (Fig. 5) three individual cathodes are arranged around a powder feeding tube. Opposite to the feeding tube a nozzle is positioned, acting as a common anode for all there cathodes. Plasma gas is fed from behind the cathode. After ignition three individual arcs are established into the center of which the powder is inserted, molten and accelerated towards the exit of the anode nozzle and subsequently the substrate.

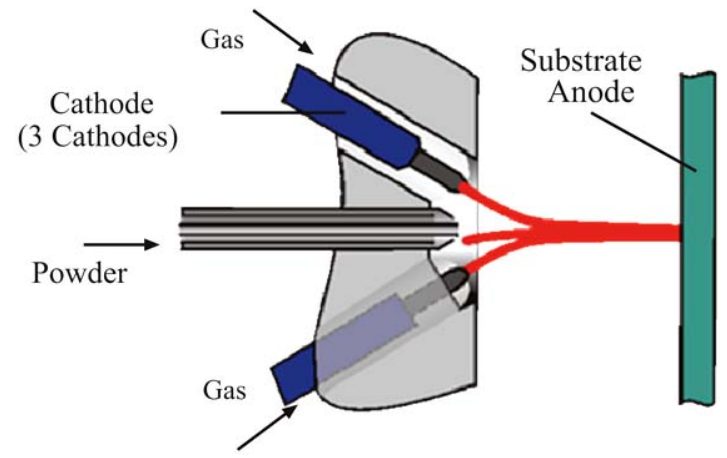

Fig. (6). Multiple cathode, single anode transferred arcs.

Another early patentable technology using multielectrodes was developed at the University of Minnesota. Even though this technology featured a transferred arc the successfull use of more than two electrodes in a single embodiement was demonstrated. A multiple cathode DC arc plasma generator arrangement is used in connection with a single anode for thermal arc plasma processing of materials (Fig. 6). A nozzle is used to introduce the gas in the center of the multiple cathodes, towards the anode. This gas flow provides control of the heat transfer to the anode and permits feeding of particles right into the core of the plasma column, which enhances inflight processing [16, 17]. This development was the first step into the direction of the socalled triple-torch reactor, consisting of three indiviual SG100 torches arranged around a feeding tube. The particulate matter or liquid exiting the feeding tube is entered into the center of the three converging plasma jets, heated and accelerated [18]. This triple-torch reactor has been used succesfully in CVD applications especially in the field of diamond synthesis [19].

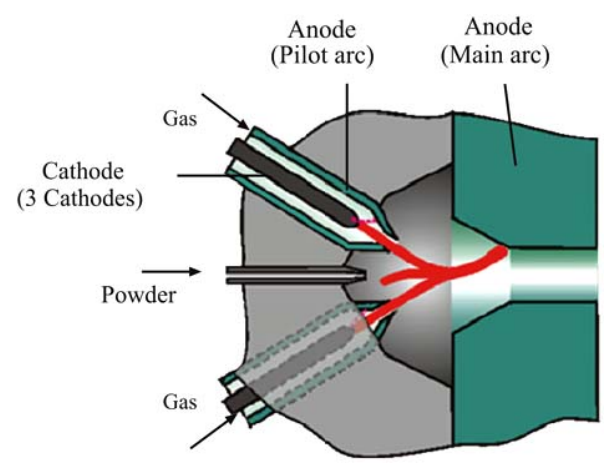

Fig. (7). Multiple cathodic attachments converging into single anode.

Marantz et al. $[20,21]$ describe a system, where the output of four plasma torches is transferred to a single anode.
The initial "conventional" plasma torches are used for ignition of the plasma only. After ignition the plasma jet exiting theses torches is transferred to a new common anode which acts as a nozzle. Similar to the triple-torch reactor the feeding tube is centered between the plasma torches, with the particulate matter heated by the converging plasma jets (Fig. 7).

At a similar time the University of British Columbia started its forray into the development of multi-electrode plasma torches [22]. In this system, a plurality of plasma guns are arranged in symmetrical relationship about a common axis along which reactant is injected through a feed pipe. This technology was the basis for the development of the Mettech Axial III, discussed below.

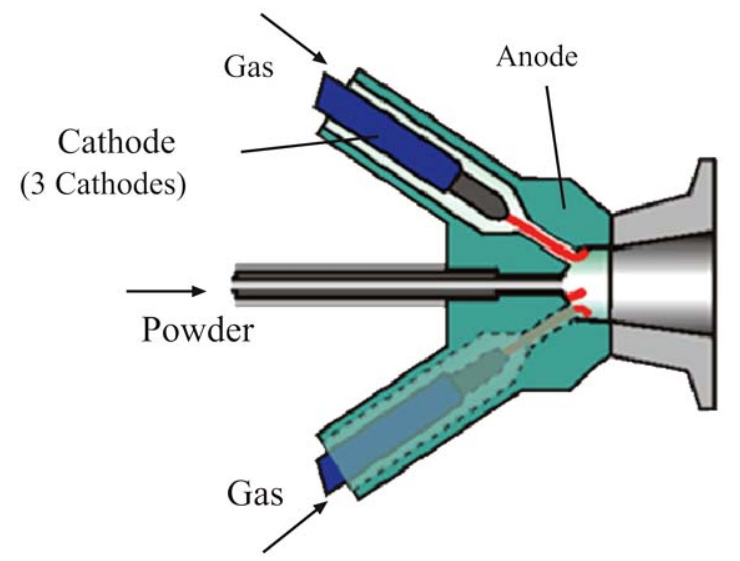

Fig. (8). Plasma torch with three cathodes, common anode embodiement.

Muehlberger et al. [23] from Electro-Plasma Inc. issued March 29, 1994 describes a converging plasma jet, which again shows some similarities to an arrangement of three individual plasma torches, except that all plasma torches are physically connected by an anode block (Fig. 8). The three plasma jets exiting from the torches are directed into a cavity which is supplied by the feeding tube and exits into a nozzle.

One of the inherent problems in all of these systems is to ensure that the reactant stream is uniformly contacted and distributed in the plasma stream formed from the converging discrete plasma jets. Another common problem in some of the systems is spitting (periodic burst of released reactant that built up in the system) which occurs when some of the reactant solidifies within the body of the converging system or block and is periodically dispersed into the plasma stream so that the flow of reactant is non-uniform. Thus none of these systems has actually had significant commercial success.

Coming back to the developments of Ross, University of British Colombia [24], this again is an embodiment, which is fairly similar to the development by Muehlberger. The main difference is the arrangement of the plasma jet, which is currently marketed and manufactured by Mettech as the Mettech Axial III [25], (Fig. 9). In order to prevent clogging an attempt was made to create plasma jets that are converged in encircling relationship about a common axis along which a reactant stream is introduced. The reactant stream is confined by the converging plasma jets, which also act as a confinement. 


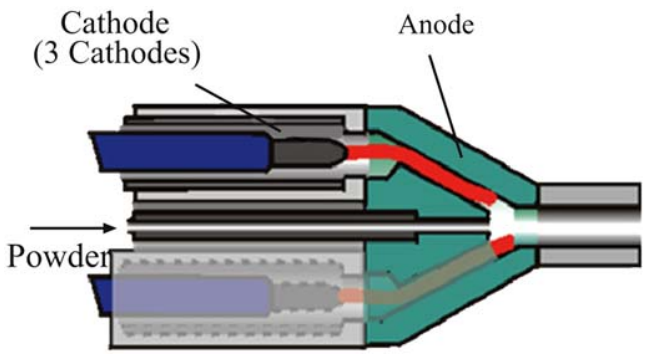

Fig. (9). Schematic of Mettech Axial III.

This torch configuration known as the Mettech Axial III System is one of the few commercially succesfull multielectrode arrangements relying on a setup of three more or less individual torches. It is used for conventional industrial coatings and high-performance applications such as graded coatings, reactive metal spraying, high temperature ceramics and spray forming. It is especially successful in connection with suspensions carrying nanoparticles [26].

Another approach to design multi-electrode plasma torches is based on the combination of experiences made with APG torch and multi-electrode setups like the one introduced by Fukanuma. The first such design is called the Triplex, initially developed at the University of the German Armed Forces in Munich (Germany) and turned into a commercial product by Sulzer Metco. Starting from the APG design the former single cathode is replaced by three water cooled tungsten cathodes of smaller diameter. These cathodes are insulated against each other and are biased separately $[27,28]$. The cathode separation is used to produce three different arcs, which produces fixed anodic arc root attachments. To prevent axial instability of the anode attachments the anode/nozzle region is divided into several thin rings all electrically insulated except for the last one which operates as a common anode (Fig. 10).

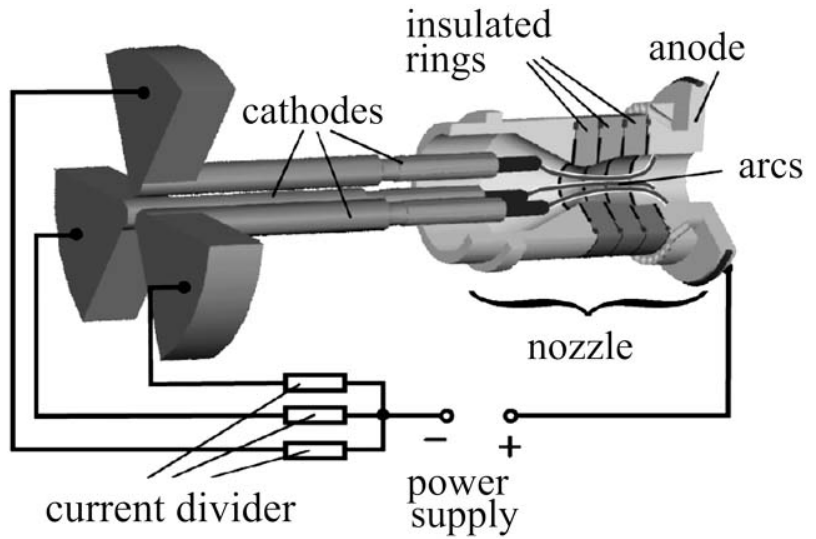

Fig. (10). Schematic of TRIPLEX plasma torch.

The variation of the cathode-anode distance by modifying the number of rings not only reduces instability, it also allows for an adjustment of the arc voltage by varying the anode/cathode distance similar to the APG. In this way the enthalpy of the plasma jet can be increased without the need of operating with molecular gases. In order to obtain three different anode attachments three individual arcs need to be produced. This is insofar difficult as the self magnetic field favours unification of the three individual arcs and thus a single anode attachment. The individual azimuthal fixation of the anodic arc roots can thus only be achieved through Steenbeck's principle of minimum enthalpy postulating a minimum arc length. Accordingly for each of the excentrically positioned cathode tips there exists one optimum length, which is the shortest connection between cathode and anode, countered by the increased cooling and therefore higher enthalpy demand the closer the arc gets to the cooled wall region.

Thus in order to produce a centered high temperature region that can lead to effective heating of the gas flow and thus produce an effective plasma jet, the arcs are forced towards each other by narrowing the nozzle diameter while still avoiding unification through the Lorentz force. As with variation of the current the attractive Lorentz force changes by the square of the current, operation in a significantly different current regime might require a variation of the nozzle diameter.

The plasma in a Triplex torch is initiated by a pilot arc in the near-cathode region, which produces sufficient charge carriers to enable an arc discharge between the cathodes and the outer anode once the anode potential has been switched electronically. The Triplex is a stable multi-electrode plasma torch, which has very much in common with regular long arc single electrode plasma torch. However, central injection has not been realized successfully, even though there is room for a powder feed tube between the cathodes, but first attempts produced significant clogging. Thus powder is fed at the exit of the nozzle, usually employing three feeding tubes. This is somewhat more efficient than powder feeding using conventional torches due to the cage effect. This effect bases on the excentric location of the three separated electric arcs, with their corresponding high temperature cores, which yields a triangular star-shaped structure of higher (at the cores) and lower (between the cores) gas viscosity. The powder injection into the central region of the plasma jet is thus more easily achieved along one of the three canals between two adjacent cores, where the gas viscosity is lower. However the powder particles do not leave by crossing through the whole plasma jet since in front of each canal the third core with its stronger axial drag is located, which causes the particles to remain within the central region. The Triplex is a commercially quite successful product, with its use extending from atmospheric plasma spraying to low pressure plasma spraying and vacuum plasma spraying [29]. One problem with the Triplex torch is the movement of the anode attachments, with changing operating conditions which displaces the preferred particle insertion points, due to the rotation of the plasma jet.

Designing a plasma torch with fixed anode attachment points resulted into development of the so-called DeltaGun, which again was developed in Munich and is currently marketed by GTV GmbH. In a DeltaGun the arc is ignited between a single cathode and three anodes (Fig. 11). The setup is again similar to the APG with an extended insulating area placed between the cathode and the anodes. The anodes are produced by dividing the anode ring (APG) into three insulated pie shaped pieces [30]. With switching of the anode potential to the three anodes a single arc is established inside the insulating region which splits up into three anode attachments near the exit. In order to protect from the thermal load as well as to avoid the oxidation of the metallic 
anodes, each anode segment is shrouded with an Argon lateral gas flow.

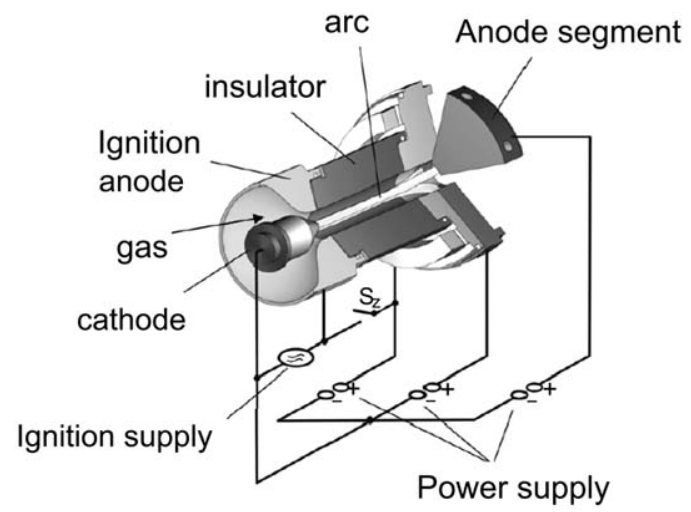

Fig. (11). Principle of operation of DeltaGun.

In similar fashion like the Triplex the anode-cathode distance can be varied, thus changing the power input and the temperature of the arc jet, produced by a strong gas flow. Similar to the Triplex a triangular structure of the arc is expected, with the cage effect providing a path for the particles to be inserted. While the anode attachment position does not change and thus the particle injecton position can be fixed, the DeltaGun suffers from slight instabilities due to axial fluctuations of the arc separation point. So far the DeltaGun has not found widespread application.

\section{MODELING}

Producing a complete numerical model for thermal plasma spray torches has long been the subject of intensive research in the thermal spray community. The numerical modeling of the gas flow and temperature distribution within the plasma torch allows for a better understanding of those processes taking place inside the torch and which are not accessible to direct experimental measurements. Additionally, a detailed modeling can lead to an improvement of the torch design in order to reduce the thermal load on the torch wall or to increase the heat transfer from the electric arc to the flowing gas.

Since the single-cathode single-anode design is the plasma torch which due to its simple geometry has found the widest industrial application, the main modeling effort has been until recently concentrated on such a torch geometry. It is nevertheless also one of the most complex plasma torches to be modeled, due to: a) the non fixed anodic arc root, particularly when operating with molecular gases $\left(\mathrm{N}_{2}\right.$ or $\left.\mathrm{H}_{2}\right)$; and b) the quasi periodic re-strike mode for the motion of the arc with its characteristic arc lengthening-instabilitygeneration of a second arc root closer to the cathode $[31,32]$.

For the torch modeling ten different variables have to be solved at each location within the plasma generator: the three components of the local gas velocity, the gas temperature (or its enthalpy), the mass density, the pressure, the electric potential and the three components of the magnetic potential. The equation set describing these variables are the NavierStokes equation (including the contribution by the Lorentz force density), the transport equation for the energy (incorporating the Joule heating due to the electric current flowing through the arc core, coupling thus the electromagnetic effect to the fluid dynamics), the mass continuity equation, the incompressibility/compressibility condition of the gas flow and the Maxwell equations for the electric and magnetic fields. The mentioned variables can be further extended with two scalar fields implementing the loss of momentum and energy due to turbulence (effect not very relevant to the gas flow within the torch $[32,33])$ and the electron temperature, the latter if non-equilibrium effects near to the electrodes or at the arc core fringes are to be included [34]. These equations and variables are solved for given boundary conditions, especially for a given mass flow at the inlet (with or without a swirl component) and the electric current (and in some models also the electric voltage) imposed externally on the torch.

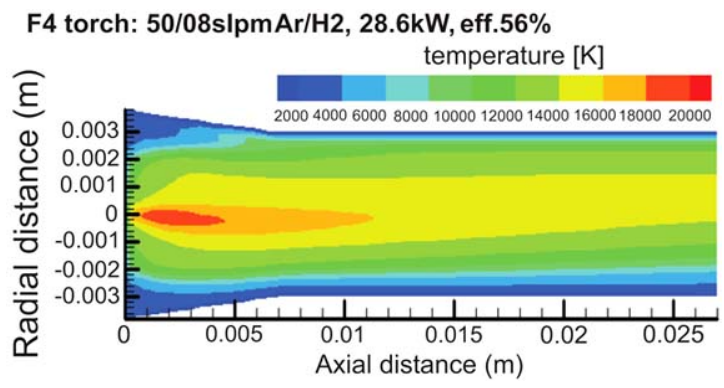

Fig. (12). Modeling of F4 torch in steady state: temperature distribution at a longitudinal section (gas flow 50/08 slpm $\mathrm{Ar} / \mathrm{H}_{2}$, current $500 \mathrm{~A}$, power $28.6 \mathrm{~kW}$ ) [33].

For the single-cathode single-anode DC torch the modeling approach has been mainly in developing a three dimensional model in steady state $[33,35]$, and only in the last five years this has been extended to a full transient simulation $[32,36,37]$ which is able to emulate the arc root motion. After various complementing efforts by different research institutions worldwide probably the most advanced description of the thermal plasma within the single-cathode single-anode torch is that of the University of Minnesota [32, 34] which also includes the separated solution of electric and heavy species temperature, incorporating some of the results derived for two-temperature transport coefficients calculated at atmospheric pressure [38].

However, due to the inherent complexity of solving the non-linear equations for the electric arc in a cross-flow of high velocity, in all the cases (steady state as well as transient) the highly non-equilibrium region very close to the cathode, including the space-charge sheath and the ionization pre-sheath, has not been implemented, unlike the more symmetric and simple transferred arc as being used in high pressure discharge lamps or in welding [39, 40]. For the plasma torches, on the contrary, it is always assumed that the gas can be described everywhere as a fluid with a local welldefined one temperature and transport coefficients (local thermodynamical equilibrium LTE, i.e. assuming all the species velocities are described by a Maxwell-Boltzmann distribution, or a slight deviations from, and ionization equilibrium holds) or in the more advanced cases by a twotemperature fluid (partial LTE). The lacking of implementing the non-equilibrium region close to the cathode, where neither the ionization equilibrium (Saha equation) nor even the quasi neutrality is fulfilled [40, 41], requires the artificial introduction of a value for the cathode 
temperature much higher than that occurring in the actual torch in order to ensure an electric conductivity high enough in front to the cathode for the arc to exist.

Further, this non-implementation of the sheath and presheath near to the cathode lets undetermined the radius of the cathode spot and thus of the radial distribution of the externally imposed electric current on the cathode surface. This missing parameter is determined either by fixing it by hand according to some estimation derived from experimental observations $[32,35]$, or by including some additional condition. In the steady state models this additional equation is the principle of minimum entropy production rate, valid for the steady state in a nonequilibrium system [42]. Alternatively, in the steady state modeling with an experimentally determined cathode spot size, the length of the electric arc, or equivalently the location of the anodic arc root, is implemented either by adjusting it to the measured torch voltage or by means of the empirical Steenbeck's principle of minimum power [35]; nevertheless this last "principle" can be shown to be actually equivalent to the principle of minimum entropy production [43] for a cylindrical symmetric transferred free-burning arc with fixed arc length, although within the single-cathode single-anode plasma torch the cylindrical symmetry and the superimposed gas flow represents an appreciable deviation from the symmetrical transferred arc [33]. As a typical example, the resulting distribution in steady state of gas temperature within a single-cathode single-anode DC torch (Sulzer Metco F4) can be seen in Fig. (12) for operation with molecular gases. It is worth noting that the validity of such a minimum principle for the steady state is a matter of discussion due to the inherent instability of the cathode spot in an axial position, as shown with a more detailed model of the interaction between the cathode and a plasma arc operating at atmospheric pressure [44, 45].

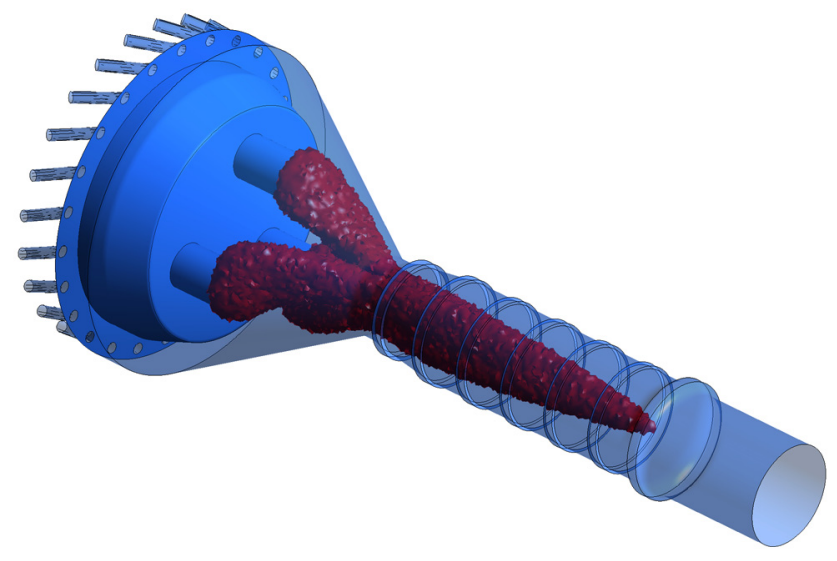

Fig. (13). Modeling of TriplexPro-200 torch in steady state: temperature iso-surface for $17000 \mathrm{~K}$ (gas flow $50 \mathrm{slpm}$ Argon, current $500 \mathrm{~A}$, power $50 \mathrm{~kW}$ ) [46].

Regarding the more advanced models, where the transient motion of the arc root along the anode wall is implemented [32, 36, 37], the size of the cathode spot is only empirically fixed and the maximal extension of the evolving arc length is determined through the incorporation of a criterion analogous to the minimum of the Paschen plot for a self substaining arc discharge: the plasma arc, lengthened by the axial gas flow, becomes instable when the local transversal electric field at some location along the fringes of the arc reaches an empirical critical value. At this situation, in the transient model, a canal of high electrical conductivity is generated connecting the anode to the arc location where the critical condition has been fulfilled. The critical electric field is so adjusted such that, combined with the imposed gas flow, it leads to a re-strike frequency close to that observed experimentally.

Based on the modeling experience with a single-cathode single-anode torch, three dimensional steady state models for a multi-electrode torch are being developed, in particular for the Triplex gun. Characteristic for the Triplex torch, the presence of three isolated electric arcs leads to a very slow convergence of the fluid dynamics equations coupled to the electric and magnetic fields generated by these arcs. Therefore, at first, only a steady state model has been attempted [46-48], which for a plasma torch operating with fixed anodic root locations has a higher validity as for the single-cathode torch with moving anode root. In all the models for the Triplex torch the same simplifications regarding the sheath and pre-sheath close to the cathode apply as for the single-cathode torch, in particular the indefinition of the cathode spot size, which has to be incorporated by hand. Of high relevance is the reported modelling result that, for the Triplex torch in its advanced design TriplexPro with a narrower torch outlet and amplified operation power, the three cathodic arcs tend to merge in a single arc inside the torch [47, 48]. Neverthelss, such modelling has been carried out for gas flows considerably higher than the usual operating conditions and thus the stated result cannot be probably applied to the typical parameters for spraying with the Triplex torch. Since the diagnostics measurement of the previous Triplex design showed a separated arrangement of the three cathodes [49] and with the aim to clarify this important issue, the TriplexPro is being modelled in the RWTH University of Aachen (Germany) as well [46], this time for current spraying parameters. In a first stage this simulation is carried out in steady state and implements in a simplified form the effect of each of the electric arcs as a heat source: in Fig. (13) the contour of a constant temperature of $17000 \mathrm{~K}$ is displayed, showing the effect of the pronounced swirl imposed on the injected gas flow at the torch inlet. The mentioned simplification regarding the heat sources as an acceptable effective implementation of the interaction between electric arc and gas flow has been observed to reproduce reasonably well the velocity and temperature distribution at least at locations near to the outlet nozzle $[47,48]$

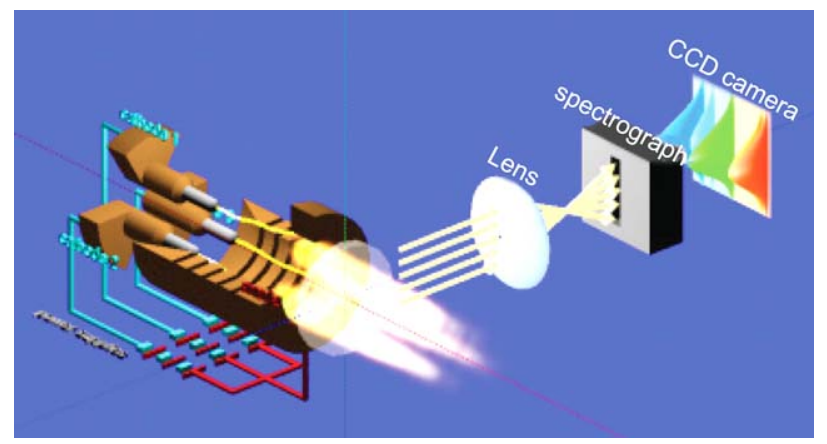

Fig. (14). Experimental setup for emission computer tomography; the camera is circling the plasma jet. 


\section{NEW DIAGNOSTIC DEVELOPMENTS}

With modeling progressing at fast rate, the need for verification is growing, especially with respect to temperature and gas velocity distribution in the jet. While progress has been made in both areas, this paper will concentrate on new results of the temperature measurement obtained with computer tomography (CT) [50].

Using CT it is possible to spectroscopically measure plasma temperatures of a non-rotationally-symmetric jet as no Abel inversion is required. For that purpose 60 individual measurements are made at 60 different angles around a half circle of the jet. Analysis of the results, however, has to rely on temporal stability of the object examined as the images are taken subsequently during a time span of a few seconds. Hence its primary application lies on the more stable multielectrode plasma torches. The principle setup for these measurements is shown in Fig. (14).

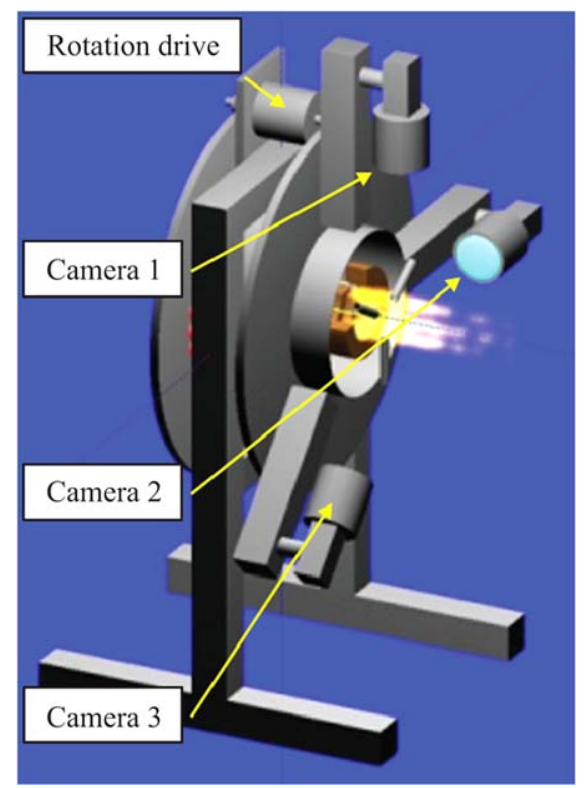

Fig. (15). Experimental setup using three CCD cameras.

Using the setup shown in Fig. (14) only a small cross section of the plasma jet can be investigated as with any regular spectrograph the $2 \mathrm{D}$ image obtained represents the intensity of the radiation entering the entrance slit versus the wavelength of the radiation. An investigation of the complete jet would therefore require numerous measurents along the axis of the plasma jet. In order to increase the field of view the CT system has been modified to allow for spectroscopic measurement without the use of a spectrograph. For that purpose the CT system was modified to use 3 individual cameras (Fig. 15) on a single turning unit. Each of these cameras is moved in $3^{\circ}$-steps $180^{\circ}$ around the plasma jet. The images obtained cover $\sim 35 \mathrm{~mm}$ along the jet axis, from which, limited by the CCD (60x60) resolution, 60 cross sections are reconstructed. The resolution is thus $0.25 \mathrm{x}$ $0.25 \mathrm{~mm}^{2}$ per pixel. In order to be able to measure plasma temperatures the three cameras were each equipped with an interference filter of either range: 1) 689-699 nm, 2) 761$771 \mathrm{~nm}$ and 3) $825-835 \mathrm{~nm}$. For every cross section an intensity distribution in the defined wavelength interval was reconstructed (Fig. 16).
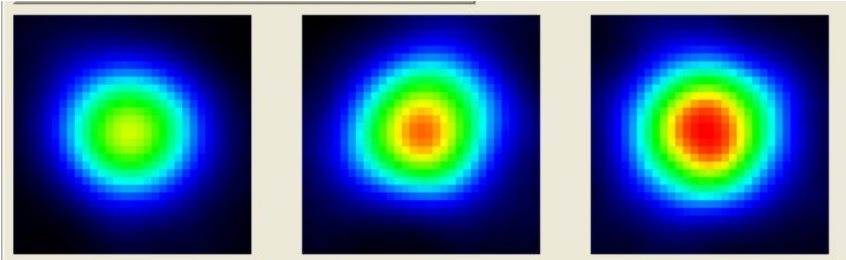

Fig. (16). DeltaGun: tomographically reconstructed cross sections of emissivity for an Argon plasma jet (gas flow 30 slpm Argon, current $340 \mathrm{~A}$, power $36 \mathrm{~kW}$ ) for three different wavelength intervals.

Using the measured ratios of intensity for each position and two wavelengths at a time, and comparing these ratios to the computed values for radiation emitted at LTE conditions, the temperature at this point can be determined. The procedure is shown in the following for the case of an Argon plasma. Assuming LTE and quasi neutrality, for a given external pressure $P$ the species densities $n(e), n(A r)$, $n\left(A r^{+}\right), n\left(A r^{++}\right)$can be determined using Saha's equation in connection with the ideal gas law [51]:

$$
\begin{aligned}
& \frac{n(e) n\left(A r^{+}\right)}{n(A r)}=\frac{2 Z\left(A r^{+}\right)}{Z(A r)}\left(\frac{2 \pi m_{e} k_{B} T}{h^{2}}\right)^{3 / 2} \exp \left(-\frac{E_{i o n-1}}{k_{B} T}\right) \equiv K_{1}(T) \\
& \frac{n(e) n\left(A r^{++}\right)}{n\left(A r^{+}\right)}=\frac{2 Z\left(A r^{+}\right)}{Z\left(A r^{+}\right)}\left(\frac{2 \pi m_{e} k_{B} T}{h^{2}}\right)^{3 / 2} \exp \left(-\frac{E_{i o n-2}}{k_{B} T}\right) \equiv K_{2}(T) \\
& \frac{P}{k_{B} T}=n(e)+n(A r)\left(1+\frac{K_{1}}{n(e)}+\frac{K_{1} K_{2}}{(n(e))^{2}}\right)=n(e)\left[1+\frac{1+\frac{K_{1}}{n(e)}+\frac{K_{1} K_{2}}{(n(e))^{2}}}{\frac{K_{1}}{n(e)}+\frac{2 K_{1} K_{2}}{(n(e))^{2}}}\right]
\end{aligned}
$$

where $m_{e}$ represents the electron mass, $T$ the temperature, $k_{B}$ Boltzmann constant, $h$ the Planck constant und $E_{\text {ion-1,-2 }}$ the first and second level of ionization of argon [52], respectively. The partition function of argon (atomic, ionized, doubly ionized) is given in (4)

$$
Z\left(A r^{(+,++)}\right)=\sum_{\text {every energy level } s} g_{A r(+,++), s} \exp \left(-\frac{E_{A r(+,++), s}}{k_{B} T}\right)
$$

whereas the contribution of the double ionization is actually negligible for the considered temperatures. Once the densities are known the spectral emissivities $\varepsilon_{\lambda, s \rightarrow d}$ can be computed [4] at each wavelength $\lambda$ for the transition of an excited state to a lower energy state $d$ :

$$
\varepsilon_{\lambda, s \rightarrow d}\left(A r^{(+)}\right)=\frac{1}{4 \pi} W_{s \rightarrow d} \frac{h c}{\lambda_{s \rightarrow d}} n\left(A r^{(+)}\right) \frac{g_{A r(+), s} \exp \left(-\frac{E_{A r(+), s}}{k_{B} T}\right)}{Z\left(A r^{(+)}\right)}
$$

where $W_{s \rightarrow d}$ is the probability [52] for such transition and $c$ represents the speed of light. The integrated emissivity within each filter interval can thus be computed assuming no doubly ionized argon is present

$$
\varepsilon_{\text {filter }}(T)=\sum_{\Delta \lambda \text { within filter }}\left(\varepsilon_{\lambda, s \rightarrow d}(A r)+\varepsilon_{\lambda, s \rightarrow d}\left(A r^{+}\right)\right)
$$

As with the measured intensities the ratio from all pairs generated by $\varepsilon_{\text {filter } 1}(T), \quad \varepsilon_{\text {filter } 2}(T)$ and $\varepsilon_{\text {filter } 3}(T)$ are 
computed over a temperature range from $5000 \mathrm{~K}$ to $25000 \mathrm{~K}$. Comparing these ratios to the measured ones allows the determination of the temperature. In order to test this setup a DeltaGun was used at fairly low power settings (current $340 \mathrm{~A}$, power $36 \mathrm{~kW}$ ). Fig. (17) shows the results of this measurement for a cross setion $\sim 1 \mathrm{~cm}$ downstream of the nozzle exit. A maximum temperature close to $14000 \mathrm{~K}$ was measured and interestingly enough no triangular structure could be observed. It might be that at the considered relative low power level, the cold and thus massive Argon flow injected laterally at the anodes is enough to "delete" the footprint of the hot three short anodic arcs.

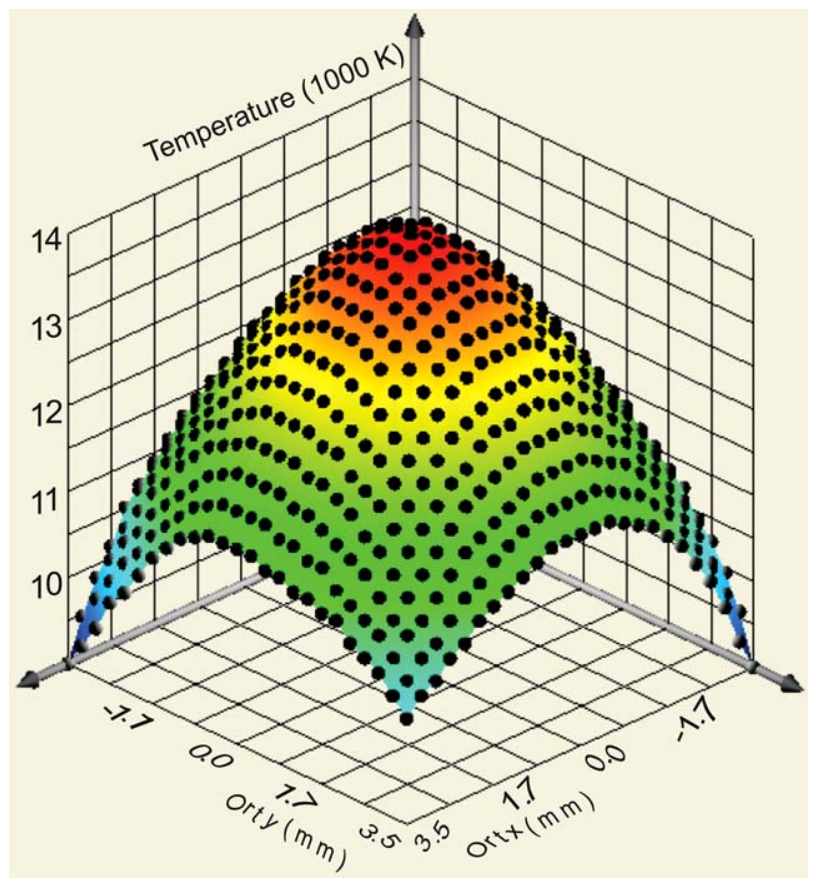

Fig. (17). Temperature profile of DeltaGun for a cross section close to nozzle (gas flow 30 slpm Argon, current 340 A, power $36 \mathrm{~kW}$ ).

This observed temperature at the plasma jet axis is compatible with a simple estimation of the radial velocity and temperature profile at the torch outlet. Due to the axial symmetry of the flow within the DeltaGun for most of its length, the velocity and temperature at the nozzle are assumed in a first approximation as having cylindrical symmetry

$v_{\text {axial }}(r)=v_{\max }\left[1-\left(\frac{r}{R}\right)^{m}\right]$ and $T(r)=T_{\max }\left[1-\left(\frac{r}{R}\right)^{n}\right]+T_{\text {wall }}$

symmetry which, according to the CT measurements just discussed, is still maintained even after the anode ring. In equation (7) $R$ denotes the nozzle radius, $T_{\text {wall }}$ is the anode wall temperature (taken here as $300 \mathrm{~K}$ ) and exponents $m=2$ and $n=4$ have been assumed, similar to those of a laminar stationary flow within a cylinder. Although the flow close to the outlet is certainly not laminar, and therefore a rather flat radial profile for velocity/temperature (large exponents $m$ and $n$ ) could be expected, due to the injection of the additional Argon shroud at the anode segments a cold layer at the plasma core fringes is to be expected near to the nozzle, and hence a more pronounced radial profile for velocity and temperature. Using the equilibrium thermodynamical plasma gas properties for Argon (density $\rho$ and enthalpy density $h$ ) listed in [4], the central velocity $v_{\max }$ and temperature $T_{\max }$ at the nozzle can be estimated to match the effective power $\dot{W}_{\text {eff }}(1)$ as well as the gas mass flow $\dot{m}$ injected into the plasma torch

$$
\begin{aligned}
\dot{m} & \approx \int_{0}^{R} \rho(T(r)) v_{\text {axial }}(r) 2 \pi r d r \\
\dot{W}_{\text {eff }} & =\int_{0}^{R} \rho(T(r)) v_{\text {axial }}(r) h(T(r)) 2 \pi r d r
\end{aligned}
$$

For the case considered, with a nozzle radius $R=3.5 \mathrm{~mm}$, a mass flow of $\dot{m}=30+20 \mathrm{slpm} \mathrm{Ar}$ (the additional $20 \mathrm{slpm}$ Ar injected at the three anodes in order to protect them) and an effective power of $\dot{W}_{\text {eff }}=0.5 \times 36 \mathrm{~kW}$ (50\% measured thermal efficiency), the resulting maximum temperature at the plasma jet axis reaches $13700 \mathrm{~K}$ (combined with a central velocity of $1850 \mathrm{~m} / \mathrm{s}$ ), close to the experimental result obtained by means of $\mathrm{CT}$.

\section{CONCLUSIONS}

The design of multi-electrode plasma torches has been an area of research for decades. Similarites in design are obvious for various embodiments, all of them with the goal to obtain better particle treatment and process stability. With the advent of new diagnostic tools such as the computer tomography and the progress of numerical simulation a better understanding of the processes in these plasma sources is expected hopefully leading to improved designs.

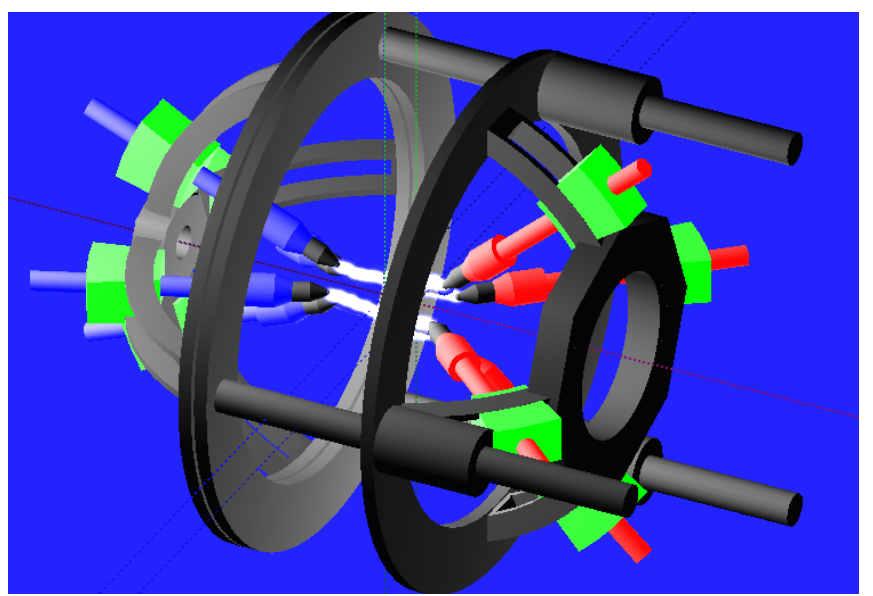

Fig. (18). Principle design of experimental multi-electrode plasma torch with varying arc lengths and electrode orientations.

\section{FUTURE WORK}

At the Laboratory for Plasma Technology of the University of the Federal Armed Forces Munich a full three dimensional model for the multi-electrode DeltaGun is currently being developed. This incorporates, on the one hand, two-temperature transport coefficients at different pressures, according to the extension in [38] of the Chapman-Enskog classical formulation. This extension allows for tracking deviations from a global LTE at the 
fringes of the plasma arc and it should, in a later stage, also allow the estimation of the plasma torch behaviour under low pressure conditions. On the other hand, the space-charge sheath as well as the ionization pre-sheath at the arc locations very close to the cathode will be implemented by means of an analytical one dimensional model in a similar way as discussed in [41]. This determines in a consistent way the cathode spot size and avoids the introduction by hand of artificially too high electrical conductivities at fluid locations close to the cathode.

On the experimental side a multi-electrode design similar to the one depicted in Fig. (18) is currently being assembled. In this equipment the relative position and orientation to each other of the three cathodes and three anodes, as well as the length of the plasma arcs, can be freely adjusted. This fully experimental torch is supposed to allow the investigation of innovative plasma stabilization methods. The interaction of multi-arc systems will be another area of research, which this setup is expected to enable.

\section{ACKNOWLEDGEMENTS}

This work was supported in part by the Deutsche Forschungsgemeinschaft (German Research Foundation), Grant SCHE 428/6-1. We appreciate the help of Jochen Zierhut for running the experimental facility and his expertise in making a Triplex and Delta work. Also we wish to thank Klaus Landes, the "father" of the Triplex and Delta for invaluable discussions.

\section{REFERENCES}

[1] Hawley D, Refke A, Landes K. Plasma plume condition forming of cascaded type plasma guns. In: Lugscheider EF, Ed. International Thermal Spray Conference Proceedings, Basel, DVS German Welding Society 2005.

[2] Fauchais P, Montavon G, Vardelle M, Cedelle J. Developments in direct current plasma spraying. Surf Coat Technol 2006; 201: 190821.

[3] Pawlowski L. The Science and Engineering of Thermal Spary Coatings. Hoboken: John Wiley \& Sons 2007.

[4] Boulos M, Fauchais P, Pfender E. Thermal Plasmas. New York: Plenum Press 1994.

[5] Chen HC, Pfender E, Heberlein J. Improvement of plasma spraying efficiency and coating quality. Plasma Chem Plasma Process 1997; 17: 93-105.

[6] Szente RN, Munz RJ, Drouet MG. Electrode erosion in plasma torches. Plasma Chem Plasma Process 1992; 12: 327-43.

[7] Chazelas C, Coudert JF, Fauchais P. Arc root behavior in plasma spray torch. IEEE Trans Plasma Sci 2005; 33: 416-7.

[8] Duan Z, Heberlein J. Arc instabilities in a plasma spray torch. J Therm Spray Technol 2002; 11: 44-51.

[9] Schein J, Zierhut J, Dzulko M, Forster G, Landes K. Improved plasma spray torch stability through multi-electrode design. Contrib Plasma Phy 2007; 47: 498-504.

[10] Heberlein J. New approaches in thermal spray technology. Pure Appl Chem 2002; 74: 327-35.

[11] Britton CR. In: Bucklow IA, Ed. 12th International Conference on Thermal Spraying Proceedings, London, Welding Institute 1989; p. 465.

[12] Vilotijevic M, Dacic B, Bozic D. Velocity and texture of a plasma jet created in a plasma torch with fixed minimal length. Plasma Sources Sci Technol 2009; 18: 1-8.

[13] Harry JE, Hobson L. Production of a Large Discharge Using a Multiple Arc System. IEEE Trans Plasma Sci 1979; 7: 157-62.

[14] Harry JE, Knight R. Simultaneous Operation of Electric Arcs from the Same Supply. IEEE Trans Plasma Sci 1981; 9: 248-54.

[15] Fukanuma H. Japanese Patent 230300 JP. 04/24/1988, 1988.
[16] Young RM, Pfender E. A Novel Approach for Introducing Particulate matter into Thermal Plasmas: The Triple-Cathode Arc. Plasma Chem Plasma Process 1989; 9: 465-81.

[17] Pfender E. Multiple arc plasma device with continuous gas jet. US patent 4818837, 1989.

[18] Asmann M, Wank A, Kim H, Heberlein J, Pfender E. Characterization of the converging jet region in a triple torch plasma reactor. Plasma Chem Plasma Process 2001; 21: 37-63.

[19] Asmann M, Cook RF, Heberlein J, Pfender E. Chemical vapor deposition of an aluminum nitride diamond composite in a triple torch plasma reactorl. J Mater Res 2001; 16: 467-77.

[20] Marantz DR, Herman H. Plasma generating apparatus and method US Patent 4982067, 1991.

[21] Marantz DR, Herman H. Plasma spray gun and method of use. US Patent 5144110, 1992.

[22] Ross DA. Plasma torch with axial reactant feed. US Patent 5008511, 1991.

[23] Muehlberger E, Muehlberger SE, Sickinger A, et al. Modular segmented cathode plasma generator. US Patent 5298835, 1994.

[24] Ross DA. Plasma jet converging system. US Patent 5556558, 1996.

[25] http://www.mettech.com/products_axial.htm

[26] Oberste Berghaus J, Marple B, Moreau C. Suspension plasma spraying of nanostructured WC-12Co coatings. J Therm Spray Technol 2006; 15: 676-81.

[27] Haslbeck P. Development of a three cathode torch applying adapted diagnostics methods. Ph.D. dissertation, thesis. Department of Electrical Enginneering, University of the Federal Armed Forces Munich (Germany) 1995; (in German).

[28] Landes K. Plasmaspritzgerät. German Patent DE 4105408 C1, $21.02,1991$.

[29] Refke A, Schmid RK. Low-pressure plasma spraying for hightemperature fuel cells. Techn Rev Sulzer (English Edition) 2005; 87: 8-10

[30] Schwenk A, Grund T, Wielage B, Zierhut J, Dzulko M, Landes K. Delta Gun - An Improved Multiple Electrode Plasma System. In: Marple BR, Hyland MM, Lau Y-C, Li C-J, Lima RS, Montavon G, Eds. International Thermal Spray Conference Proceedings, Beijing, ASM International 2007.

[31] Dorier JL, Gindrat M, Hollenstein C, Salito A, Loch M, Barbezat G. Time-Resolved Imgaing of Anodic Arc Root Behavior During Fluctuations of a DC Plasma Spraying Torch. IEEE Trans Plasma Sci 2001; 29: 494-501

[32] Trelles JP, Pfender E, Heberlein J. Modelling of the arc reattachment process in plasma torches. J Phys D Appl Phys 2007; 40: $5635-48$.

[33] Ramachandran K, Marqués JL, Vaßen R, Stöver D. Modelling of arc behaviour inside a F4 APS torch. J Phys D Appl Phys 2006; 39: 3323-31.

[34] Trelles JP, Heberlein J, Pfender E. Non-equilibrium modellling of arc plasma torches. J Phys D Appl Phys 2007; 40: 5937-52.

[35] Li HP, Pfender E, Chen X. Application of Steenbeck's minimum principle for three-dimensional modelling of DC arc plasma torches. J Phys D Appl Phys 2003; 36: 1084-96.

[36] Baudry C. Contribution to the transient and three dimensional modeling of the arc dynamic behaviour in a plasma torch", Ph.D. dissertation. Department of Ceramics Processing, Limoges University. (France) 2003; (in French).

[37] Baudry C, Vardelle A, Mariaux G. Numerical modeling of a DC non-transferred plasma torch: Movement of the arc anode attachment and resulting anode erosion. High Technol Plasma Process 2005; 9: 1-15.

[38] Rat V, André P, Aubreton J, Elchinger MF, Fauchais P, Lefort A. Transport properties in a two-temperature plasma: Theory and application. Phys Rev E 2001; 64: 026409-1-20

[39] Zhou X, Heberlein J. Analysis of the arc-cathode interaction of free-burning arcs. Plasma Sources Sci Technol 1995; 2: 564-74.

[40] Wendelstorf J. Ab-inition modeling of thermal plasma gas discharges (electric arcs). Ph.D. dissertation. Dept. Physics, Braunschweig Univ. (Germany) 2000; (in English).

[41] Benilov MS, Marotta A. A model of the cathode region of atmospheric pressure arcs. J Phys D Appl Phys 1995; 28: 1869-82.

[42] DeGroot SR, Mazur P. Non-equilibrium Thermodynamics. Amsterdam: North Holland 1969.

[43] Peters T. Über den Zusammenhang des Steenbeckschen Minimumprinzips mit dem thermodynamischen Prinzip der minimalen Entreopieerzeugung. Z Phys A 1956; 144: 612-31. 
[44] Benilov MS. Stability of direct current transfer to thermionic cathodes: I. Analytical theory. J Phys D Appl Phys 2007; 40: 137693.

[45] Benilov MS, Faria MJ. Stability of direct current transfer to thermionic cathodes: II. Numerical simulation. J Phys D Appl Phys 2007; 40: 5083-97.

[46] Bobzin K, Bagcivan N, Parkot D, et al. Homogenization of coating properties in Atmospheric Plasma Spraying - New results of a DFG (German Research Foundation)-funded research group. In: Marple BR, Hyland MM, Lau Y-C, Li C-J, Lima RS, Montavon G, Eds. International Thermal Spray Conference Proceedings, Las Vegas, ASM International 2009.

[47] Muggli F, Molz R, McCullogh R, Hawley D. Improvement of Plasma Gun Performance using Comprehensive Fluid Element Modeling: part I. In: Marple BR, Hyland MM, Lau Y-C, Li C-J, Lima RS, Montavon G, Eds. International ThermSpray Conference Proceedings, Beijing, ASM International 2007; pp. 146-51.

[48] Molz R, McCullogh R, Hawley D, Muggli F. Improvements of Plasma Gun Performance using Comprehensive Fluid Element
Modeling II. In: Marple BR, Hyland MM, Lau Y-C, Li C-J, Lima RS, Montavon G, Eds. International ThermSpray Conference Proceedings, Beijing, ASM International 2007; pp. 152-7.

[49] Landes K, Forster G, Zierhut J, Dzulko M, Hawley D. Computer Tomography of Plasma Jets - Applied on a Triplex II torch. International ThermSpray Conference Proceedings, Osaka, DVS Verlag 2004

[50] Schein J, Richter M, Landes K, Forster G, Zierhut J, Dzulko M. Tomographic Investigation of Plasma Jets Produced by Multielectrode Plasma Torches. J Therm Spray Technol 2008; 17: 338-43.

[51] Drellishak KS, Knopp CF, Bulent Cambel A. Partition Functions and Thermodynamic Properties of Argon Plasma. Phys Fluids 1963; 6: 1280-8.

[52] http://physics.nist.gov/PhysRefData/ASD/levels_form.html (energy levels). And http://physics.nist.gov/PhysRefData/ASD/lines form. html (transition probabilities).

(C) Marqués et al.; Licensee Bentham Open.

This is an open access article licensed under the terms of the Creative Commons Attribution Non-Commercial License (http://creativecommons.org/licenses/by$\mathrm{nc} / 3.0 /$ ) which permits unrestricted, non-commercial use, distribution and reproduction in any medium, provided the work is properly cited. 